\title{
SPOrt: an Experiment Aimed at Measuring the Large Scale Cosmic Microwave Background Polarization
}

\author{
Ettore Carretti, ${ }^{a}$ Stefano Cortiglioni, ${ }^{a}$ Gianni Bernardi, ${ }^{a}$ Stefano Cecchini, ${ }^{a}$ \\ Claudio Macculi, ${ }^{a}$ Carla Sbarra, ${ }^{a}$ Jader Monari, ${ }^{b}$ Alessandro Orfei, ${ }^{b}$ Marco Poloni, ${ }^{b}$ \\ Sergio Poppi ${ }^{b}$ Giuliano Boella ${ }^{c}$ Silvio Bonometto,${ }^{c}$ Massimo Gervasi,${ }^{c}$ Giorgio Sironi,${ }^{c}$ \\ Mario Zannoni, ${ }^{d}$ Marco Tucci, ${ }^{e}$ Massimo Baralis, ${ }^{f}$ Oscar A. Peverini, ${ }^{f}$ Riccardo Tascone,${ }^{f}$ \\ Giuseppe Virone,${ }^{f}$ Roberto Fabbri, ${ }^{g}$ Luciano Nicastro, ${ }^{h}$ Kin-Wang Ng,${ }^{i}$ V.A. Razin,${ }^{j}$ \\ Evgenij N. Vinyajkin, ${ }^{j}$ Mikhail V. Sazhin, ${ }^{k}$ Igor A. Strukov ${ }^{l}$ \\ ${ }^{a}$ IASF/CNR Sezione di Bologna, Via Gobetti 101, I-40129 Bologna, Italy \\ ${ }^{b}$ IRA/CNR, Via Gobetti 101, I-40129, Bologna, Italy \\ ${ }^{c}$ Dip. di Fisica, Univ. di Milano - Bicocca, P.za della Scienza 3, I-20126 Milano, Italy \\ ${ }^{d}$ IASF/CNR Sezione di Milano, Via Bassini 15, I-20133 Milano, Italy \\ ${ }^{e}$ Instituto de Fisica de Cantabria, Avda Los Castros s/n, 39005 Santander, Spain

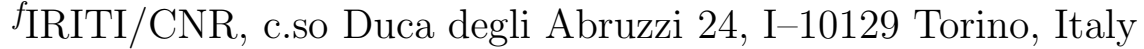 \\ ${ }^{g}$ Dip. di Fisica, Univ. di Firenze, Via Sansone 1, I-50019 Sesto Fiorentino (FI), Italy \\ ${ }^{h}$ I.A.S.F./C.N.R. Sez. di Palermo, via U. La Malfa 153, I-90146 Palermo, Italy \\ ${ }^{i}$ Academia Sinica, 11529 Taipei, Taiwan \\ ${ }^{j}$ NIRFI, 25 B.Pecherskaya st., Nizhnij Novgorod 603600/GSP-51, Russia \\ ${ }^{k}$ Shternberg Astronomical Institute, Moscow State University, Moscow 119992, Russia \\ ${ }^{l}$ IKI, Profsojuznaja ul. 84/32, Moscow 117810, Russia
}

\begin{abstract}
SPOrt (Sky Polarization Observatory) is a space experiment to be flown on the International Space Station during Early Utilization Phase aimed at measuring the microwave polarized emission with $\mathrm{FWHM}=7^{\circ}$, in the frequency range 22-90 GHz. The Galactic polarized emission can be observed at the lower frequencies and the polarization of Cosmic Microwave Background $(\mathrm{CMB})$ at $90 \mathrm{GHz}$, where contaminants are expected to be less important. The extremely low level of the CMB Polarization signal $(<1 \mu \mathrm{K})$ calls for intrinsically stable radiometers. The SPOrt instrument is expressly devoted to CMB polarization measurements and the whole design has been optimized for minimizing instrumental polarization effects. In this contribution we present the receiver architecture based on correlation techniques, the analysis showing its intrinsic stability and the custom hardware development carried out to detect such a low signal.
\end{abstract}

Keywords: CMB, Polarization, Polarimeter, Instrumental Effects

\section{INTRODUCTION}

The Cosmic Microwave Background (CMB) is a powerful tool for understanding the origin and evolution of the Universe. The hot Big Bang model predicts the CMB is a Black Body radiation almost isotropic and unpolarized. Any deviations from this ideal behaviour is related to cosmological parameters and allow their determination. ${ }^{1-4}$ Very small CMB Anisotropies (CMBA) has been detected at both $\operatorname{large}^{5,6}$ and small ${ }^{7-9}$ angular scales, but only upper limits on the CMB Polarization (CMBP) have been established up to now. In

Further author information: (Send correspondence to E.C.)

E.C.: E-mail: carretti@bo.iasf.cnr.it, Telephone: +39 0516398735 

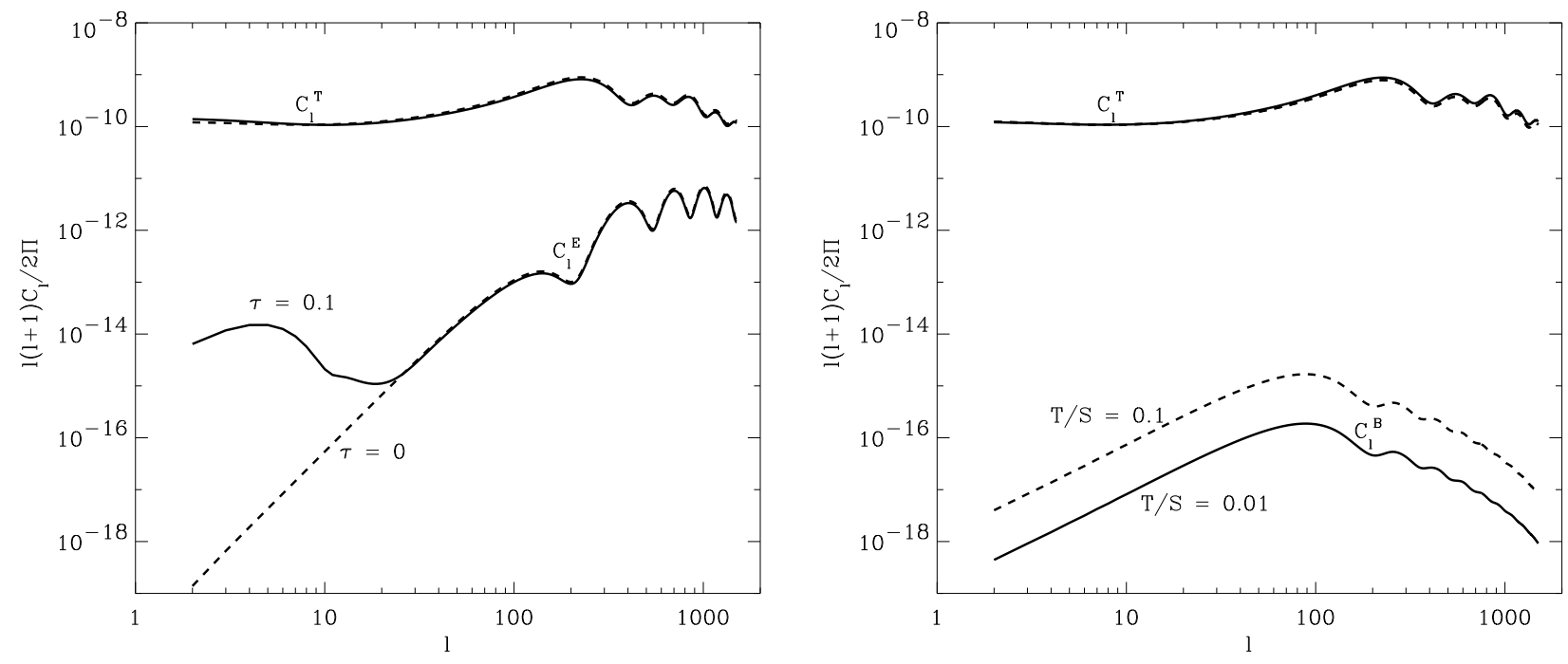

Figure 1. Left: Temperature anisotropy and E-mode power spectra. Two $\Lambda$ CDM $\left(\Omega_{\Lambda}=0.7\right)$ cosmological models which differ only in the re-ionization optical depth $\tau$ are shown. Right: Temperature anisotropy and B-mode power spectra. Two $\Lambda \mathrm{CDM}\left(\Omega_{\Lambda}=0.7\right)$ cosmological models which differ only in the tensor (gravitational waves) to scalar perturbation ratio $\mathrm{T} / \mathrm{S}$ are shown.

spite of its elusiveness, the CMBP promises to add information to the CMBA data: it will provide a direct measurement of cosmological parameters that CMBA alone is not able to determine. ${ }^{3}$ The optical depth $\tau$ in the dark ages and the epoch $z_{\text {ri }}$ at which the re-ionization occurred are directly measured by CMBP providing us with the formation epoch of the first structures and their growth-rate. Figure 1 presents a comparison between temperature and polarization (E-mode) power spectra for two cosmological models, which differ only in the optical depth $\tau$ of the re-ionized medium. It is clear that the E-mode spectrum is much more sensitive to $\tau$ than the temperature spectrum and that this new information is found at large angular scales $(l<10$, i.e. $\left.\theta>20^{\circ}\right)$. In addition, the E-mode of the CMBP brings important information also at subdegree angular scales, where the coherent primordial fluctuations predicted by inflation leave fingerprints like a well defined Doppler peak pattern: the peaks in the $\mathrm{T}$ spectrum correspond to minima in the E spectrum and viceversa. Thus, the detection of the CMBP at subdegree scales leads to an indirect check of the inflationary model. ${ }^{10}$

The detection of the B-mode is even more exciting, although the signal is very weak: its level is directly related to the tensor-to-scalar perturbation ratio T/S (see Figure 1), whose value is in turn related to the energy of the Universe at the inflation time. ${ }^{11,12}$ Thus, the measurement of the B-mode allows the estimate of the energy at which the inflation occurred and the identification of the right model in the zoo of the existing ones.

Moreover, besides their intrinsic interest, $\tau, z_{\mathrm{ri}}$ and $\mathrm{T} / \mathrm{S}$ determinations further improve the precision on other cosmological parameters.

Unfortunately, the CMBP predicted level is very low (few $\mu \mathrm{K}$ on sub-degree scales and less than $1 \mu \mathrm{K}$ on large scales). Current experimental upper limits are still one order of magnitude higher than the predicted level ${ }^{13-23}$ and, even though more sensitive detectors are coming, CMBP measurements are biased by foreground subtraction. Besides its intrinsic interest, the Galaxy acts as a foreground for CMB experiments and only its accurate knowledge will allow measurements of CMB features (See Ref. 24-26 and references therein). So far, polarization surveys have been carried out only at frequencies up to $2.7 \mathrm{GHz},{ }^{27-31}$ where the Galactic emission appeares to be dominated by synchrotron. Such observations either are widely undersampled ${ }^{27}$ or cover narrow stripes around the Galactic Plane. ${ }^{28-31}$ A better estimate of the foreground contaminations can be done only through Galactic surveys at frequencies closer to the range of interest of CMBP measurements. 


\section{THE SPORT EXPERIMENT}

The purpose of Sky Polarization Observatory (SPOrt)* is aimed at filling the current gap in measurements of the diffuse polarized emission in the 22-90 GHz range. Together with BaR-SPOrt, on-ground observations and technological activities, it is part of the SPOrt Programme ${ }^{32}$ aimed at detecting the CMBP. SPOrt is an Italian Space Agency (ASI) funded experiment and it has been selected by ESA to be flown onboard the International Space Station (ISS) for a minimum lifetime of 18 months, starting from 2005 (see Figure 2).

Figure 2. SPOrt position on the External Payload Facility of Columbus onboard the ISS (courtesy by Alenia Spazio).

SPOrt is the first space instrument devoted to $Q \& U$ Stokes parameters measurements in the microwave domain at large angular scales $\left(\theta>7^{\circ}\right)$. This can be done only by all-sky surveys (space experiments) and by instruments designed to be as much as possible insensitive to instrumental polarization.

The main features of SPOrt are the following (see also Table 1):

- multifrequency approach with four frequency channels at 22,32, 60 and $90 \mathrm{GHz}$ to match the best band for CMBP observation $(90 \mathrm{GHz})$, while checking the Galactic contributions (22-90 GHz) and mapping the Galactic synchrotron emission (22 and $32 \mathrm{GHz}$ ).

- very simple optics (corrugated feed horns), providing angular resolutions down to $7^{\circ}$, suitable to access the new information on cosmological parameters contained in CMBP on large scales, while minimizing optics systematic effects.

- a nearly all-sky survey ( 80\% sky coverage).

Table 1. SPOrt main features: $N_{P X}$ is the number of FWHM pixels covered by SPOrt, $\sigma_{1 s}$ is the istantaneous sensitivity (1 second), $\sigma_{P X}$ and $\sigma\left(P_{\mathrm{rms}}\right)$ are the final sensitivity per pixel and for the $P_{\mathrm{rms}}=\sqrt{\left\langle Q^{2}+U^{2}\right\rangle}$, respectively, considering a 18 month mission lifetime and $50 \%$ observing efficiency.

\begin{tabular}{ccccccccc}
\hline$\nu[\mathrm{GHz}]$ & BW & FWHM & Orbit Time $[\mathrm{s}]$ & Coverage & $N_{P X}$ & $\sigma_{1 s}\left[\mathrm{mKs}^{1 / 2}\right]$ & $\sigma_{P X}[\mu \mathrm{K}]$ & $\sigma\left(P_{\mathrm{rms}}\right)[\mu \mathrm{K}]$ \\
\hline $22,32,60,90$ & $10 \%$ & $7^{\circ}$ & 5400 & $80 \%$ & 660 & 1.0 & 5.2 & 0.3 \\
\hline
\end{tabular}

${ }^{*}$ http://sport.bo.iasf.cnr.it 
The CMBP signal is weak (about 1-10\% of CMBA, depending on the scale) requiring expressly devoted instruments, as CMBA does. Thus, great care has been taken to optimize the instrument design with respect to systematics generation, long term stability and observing time efficiency.

The following major choices were adopted for the SPOrt design:

1. correlation polarimeters to improve the stability (see Figure 3);

2. correlation of the two circularly polarized components $E_{L}$ and $E_{R}$ to directly and simultaneously measure both $Q$ and $U$ (100\% observing time efficiency)

$$
\begin{aligned}
Q & \propto \Re\left(E_{R} E_{L}^{*}\right) \\
U & \propto \Im\left(E_{R} E_{L}^{*}\right)
\end{aligned}
$$

This optimizes the sensitivity with respect to other schemes which provide either $Q$ or $U$ at once, just resulting in 50\% efficiency (e.g. correlation or difference of the linear components);

3. detailed analysis of the correlation scheme to minimize the instrumental systematics by the identification of "critical" components and the specifications they have to satisfy;

4. custom development of these components when the existing state-of-the-art is not enough.

\section{DESIGN ANALYSIS}

The radiometer equation ${ }^{33,34}$ helps us find the parameters to be controlled for minimizing systematic effects. In fact, in the expression ${ }^{\dagger}$

$$
\Delta T_{\mathrm{rms}}=\sqrt{\frac{k^{2} T_{\mathrm{sys}}^{2}}{\Delta \nu \tau}+T_{\text {offset }}^{2}\left(\frac{\Delta G}{G}\right)^{2}+\Delta T_{\text {offset }}^{2}}
$$

the first term represents the white noise of an ideal and stable radiometer, while the second and the third terms are the gain and offset fluctuation effects, respectively, and represent the additional noise generated by instrument instabilities. The ideal behaviour is preserved provided that the offset is kept under control.

Correlation receivers are intrinsically more stable because of their lower offset generation. Figure 3 shows the scheme we adopted for the SPOrt radiometers. Polarizer and OMT extract the two circularly polarized components collected by a dual-polarization feed horn. After amplification, the two components are correlated by the correlation unit (CU). The latter includes an Hybrid Phase Discriminator (HPD), diodes and differential amplifiers, whose outputs are the two Stokes parameters $Q \& U$.

In order to minimize the offset level an analysis has been carried out to identify the devices generating offset sources and the parameters to be controlled. The analysis shows the offset is generated at both CU and antenna system (horn, polarizer and OMT) levels.

The CU needs an HPD with high rejection of the unpolarized components. This has been achieved by the development of a custom device ${ }^{35,36}$ providing $>30 \mathrm{~dB}$ rejection. In combination with a lock-in system, this makes the $\mathrm{CU}$ contribution to the offset negligible, the total rejection being $>60 \mathrm{~dB}$.

Consequently, the antenna system is the most important offset source. Carretti et al. in Ref. 37 found that: $¥$

$$
T_{\text {offset }}=S P_{\mathrm{OMT}}\left(T_{\text {sky }}+T_{\text {noise }}^{\mathrm{Ant}}\right)+S P_{\mathrm{pol}}\left(T_{\text {sky }}+T_{\text {noise }}^{\mathrm{horn}}-\frac{T_{\mathrm{ph}}^{\mathrm{pol}}}{\eta}\right),
$$

\footnotetext{
${ }^{\dagger} T_{\text {sys }}, T_{\text {offset }}$ and $\Delta T_{\text {offset }}$ are the system temperature, the offset equivalent temperature and its fluctuation, respectively; $G$ is the radiometer gain, $\tau$ the integration time, $\Delta \nu$ the radiofrequency bandwidth and $k$ a constant depending on the radiometer type.

${ }^{\ddagger} T_{\text {sky }}$ is the signal collected from the sky, $T_{\text {noise }}^{\text {horn }}$ is the noise generated by the horn only, $T_{\text {noise }}^{\text {Ant }}$ is the noise temperature by the whole antenna system, $\eta$ is the efficiency of the feed horn and $T_{\mathrm{ph}}^{\mathrm{pol}}$ is the physical temperature of the polarizer.
} 


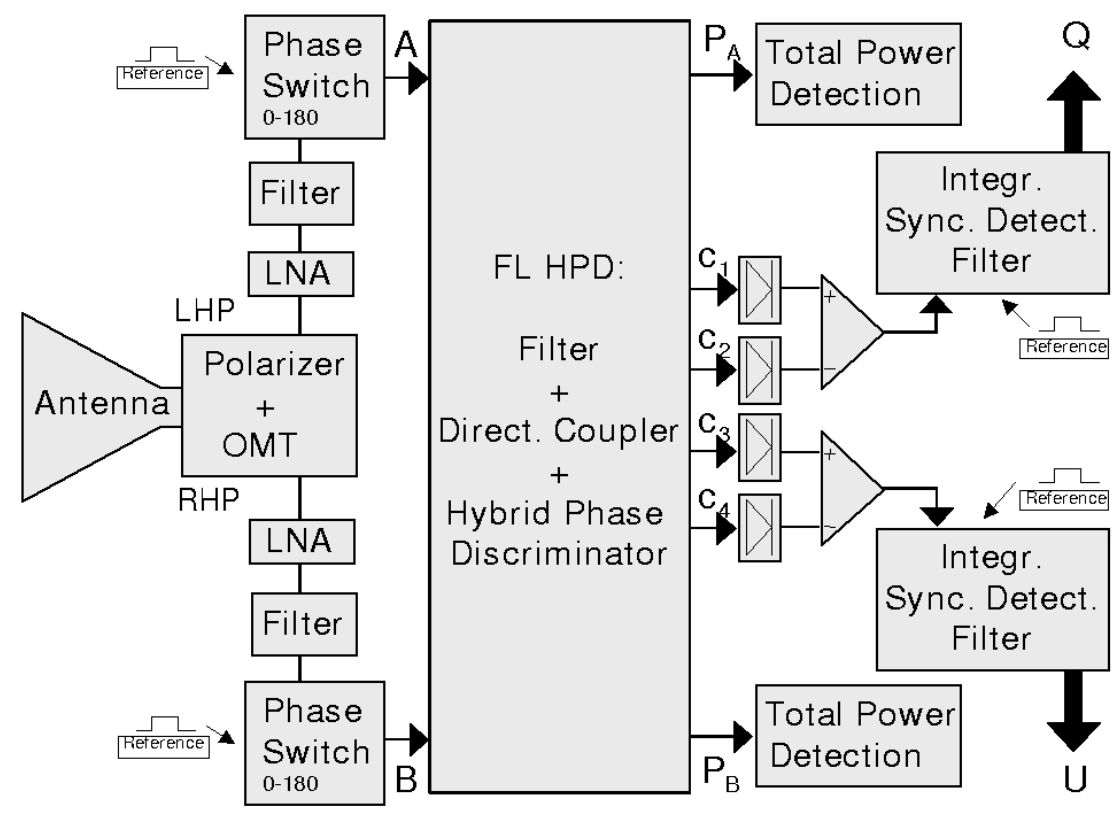

Figure 3. Schematic block diagram of the SPOrt radiometers. Polarizer and OMT extract the two circularly polarized components LHP \& RHP collected by the horn. After amplification, the correlation unit (based on a Hybrid Phase Discriminator, see ${ }^{35,36}$ for details) provides directly both $Q \& U$.

where the two quantities

$$
\begin{aligned}
S P_{\mathrm{OMT}} & =2 \frac{\Re\left(S_{A 1} S_{B 1}^{*}\right)}{\left|S_{A 1}\right|^{2}}, \\
S P_{\mathrm{pol}} & =\frac{1}{2}\left(1-\frac{\left|S_{\perp}\right|^{2}}{\left|S_{\|}\right|^{2}}\right),
\end{aligned}
$$

describe the goodness of the OMT and of the polarizer, respectively, from the offset generation point of view. Uncorrelated signals (noise and sky) are partially detected as correlated signals because of the OMT cross-talk ( $S_{A 1}$ and $S_{B 1}$ are the transmission and cross-talk coefficient of the OMT, respectively) and of the polarizer attenuation difference $\left(S_{\|}\right.$and $S_{\perp}$ are the attenuations of the two polarization of the polarizer).

The instability of a radiometer can be measured in terms of the knee frequency $\left(f_{\text {knee }}\right)$, that provides the time scale at which the $1 / f$ component of the noise power spectrum prevails on the white noise. Destriping techniques can remove most of the effects of the $1 / f$ noise, but only if the knee frequency is lower than the signal modulation frequency. ${ }^{38}$ For SPOrt this corresponds to the orbit frequency $f_{\text {orbit }}=1.8 \times 10^{-4} \mathrm{~Hz}$.

Currently available InP Low Noise Amplifiers have rather high knee frequencies $\left(f_{\mathrm{knee}}^{\mathrm{lna}} \sim 100-1000 \mathrm{~Hz}\right)$, making correlation architectures more convenient. In fact, the knee frequency of a correlation receiver is related to that of its amplifiers by the formula

$$
f_{\text {knee }}=\left(\frac{T_{\text {offset }}}{T_{\text {sys }}}\right)^{2} f_{\text {knee }}^{\text {lna }}
$$

where $T_{\text {offset }}$ is the radiometric offset and $T_{\text {sys }}$ is the system temperature.

Equations (3)-(5) state that the main offset sources are the OMT cross-talk and the difference between the attenuations of the two polarizations of the polarizer. SPOrt needs have been quantified in $-60 \mathrm{~dB}$ and $-30 \mathrm{~dB}$, 
respectively, leading to an offset value as low as $T_{\text {offset }} \sim 50 \mathrm{mK}$ which, combined with a $T_{\text {sys }} \sim 100 \mathrm{~K}$, gives the knee frequency

$$
f_{\text {knee }} \sim 2.5 \times 10^{-7} f_{\text {knee }}^{\text {lna }}
$$

matching the condition for a succesful destriping $\left(f_{\text {knee }}<f_{\text {orbit }}\right)$

However, state-of-the-art OMTs are not good enough, and custom hardware development has been required to the SPOrt team. Figure 4 shows the result obtained for the $32 \mathrm{GHz}$ channel: a cross-talk as low as $-65 \mathrm{~dB}$ has been achieved, well matching the $-60 \mathrm{~dB}$ goal.

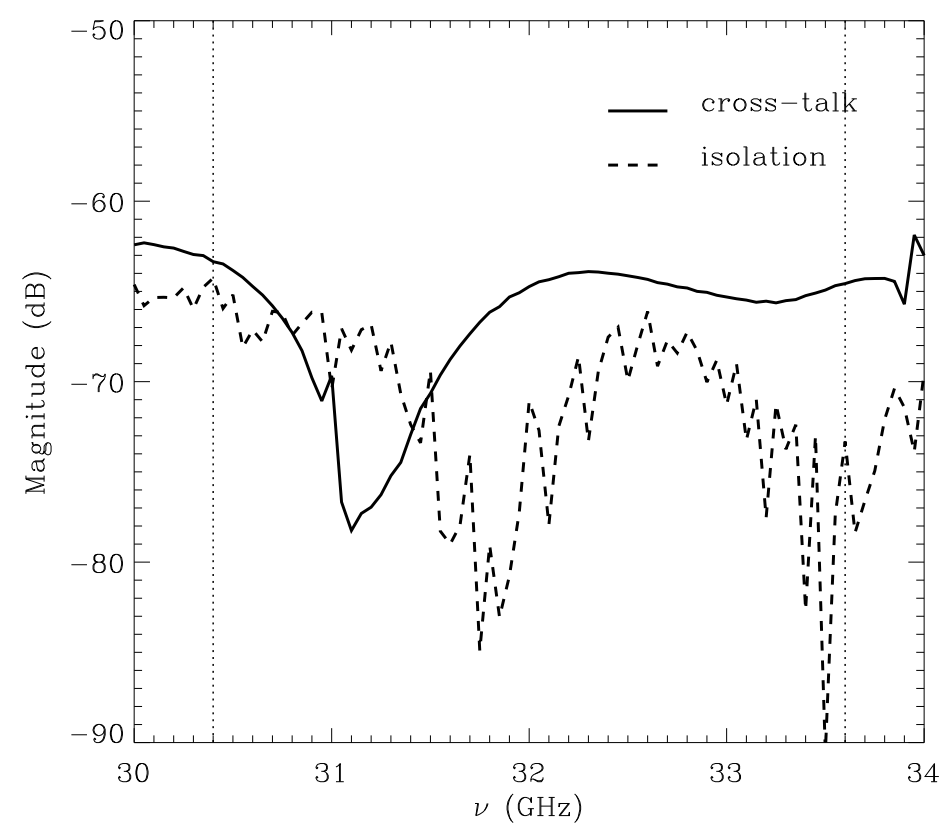

Figure 4. Magnitudes of both the isolation between the two rectangular ports and the cross-talk between the two polarizations for the $32 \mathrm{GHz}$ SPOrt OMT. The vertical dotted lines show the $10 \%$ band.

Besides the offset generation, the SPOrt team has identified another systematic error source: the spurious polarization generated by the optics. ${ }^{37}$ This is due to the anisotropy distribution of the unpolarized radiation modulated by the $f$ pattern:

$$
\begin{aligned}
& T^{\text {horn }}= \frac{1}{\Omega_{A}} \int_{0}^{\pi} \sin \theta d \theta \int_{0}^{\pi / 2} d \phi\left[\Delta T_{b}(\theta, \phi)-\Delta T_{b}(\theta, \phi+\pi / 2)+\right. \\
&\left.\Delta T_{b}(\theta, \phi+\pi)-\Delta T_{b}(\theta, \phi+3 / 2 \pi)\right] \cdot f(\theta, \phi), \\
& f(\theta, \phi)=-P(\theta, \phi) \chi^{*}(\theta, \phi+\pi / 2)+\chi(\theta, \phi) P^{*}(\theta, \phi+\pi / 2),
\end{aligned}
$$

where $P$ and $\chi$ are the co-polar and cross-polar patterns, respectively, and $\Omega_{A}$ is the antenna beam. In the case of SPOrt feed horns, the contribution of the $f$ pattern is $\sim-24 \mathrm{~dB}$ and the rms contamination from the $30 \mu \mathrm{K}$ of the CMB anisotropy is lower than $0.2 \mu \mathrm{K}$. Due to its intrinsic asimmetry, off-axis optics with the same cross-polar pattern level would imply a spurious contribution 8-10 dB higher.

Moreover, also the hardware calibration of a CMBP experiment represents a challenge. Standard marker injectors are not suitable for calibrating a tensorial quantity as the pair $(Q, U)$. Thus, a new concept calibrator has been developed, valid for any radio-polarimeter, based on the insertion of three signals at different position angles. This device is similar to that of BaR-SPOrt and further details can be found in Ref. 39, 40 . 
In summary, the faint CMBP signal requires specifically devoted instruments and the SPOrt team has spent (and is still spending) a big effort in designing an instrument with very low systematic error contamination, characterized by:

- correlation unit with high rejection of the unpolarized component ( $>60 \mathrm{~dB}$ ) based on a custom-developed HPD and a lock-in system;

- on axis and simple optics (corrugated feed horns) in order to minimize the spurious polarization induced by both the $f$ pattern and the CMB temperature anisotropy at the beam scale: With such a configuration $\sim-35 \mathrm{~dB}$ of cross-polarization translates into a contamination $<0.2 \mu \mathrm{K}$;

- high OMT isolation $(>60 \mathrm{~dB})$ and low cross-talk $(<-60 \mathrm{~dB})$, since these parameters are among the major responsibles for $Q \& U$ offset generation in correlation polarimeters;

- very small difference $(<-30 \mathrm{~dB})$ between the attenuations of the two polarizations in the polarizer, which is the other main responsible of offset generation.

\section{SCIENTIFIC GOALS}

The goals of SPOrt are essentially two:

- to provide $7^{\circ}$ (FWHM) full maps of the Galactic synchrotron emission at 22-32 GHz. The diffuse Galactic polarization, at frequencies greater than $2.7 \mathrm{GHz}$, is practically unknown. However, a level of $T_{\text {syn }}(30 \mathrm{GHz}) \sim 5 \mu \mathrm{K}$ on $7^{\circ}$ scales can be evaluated by down extrapolating data from Duncan et al.. ${ }^{28}$ Unpolarized data may provide an independent confirmation assuming, for example, a $30 \%$ of polarization in the COBE-DMR maps.

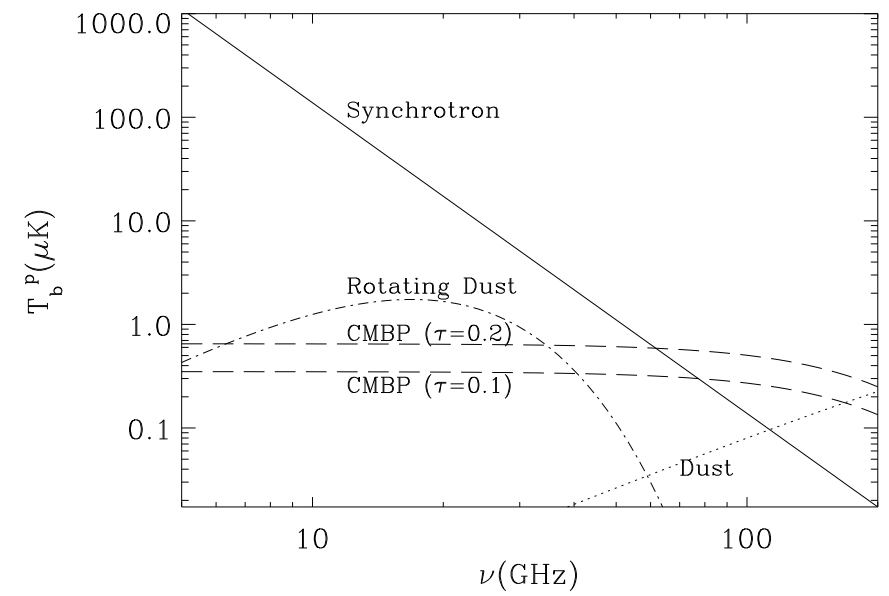

Figure 5. Expected polarized brightness temperature for the relevant polarized Galactic foregrounds on $7^{\circ}$ scale. The synchrotron emission has been normalized to $T_{\mathrm{syn}}(30 \mathrm{GHz}) \sim 5 \mu \mathrm{K}$ : See text for details. The parameters of the other components are from Tegmark et al.. ${ }^{25}$ The CMBP behaviour for two $\Lambda$ CDM models $\left(\Omega_{\Lambda}=0.7\right)$ with optical depth $\tau=0.1$ and $\tau=0.2$ are also shown.

The expected scenario is sketched in Figure 5, where the CMBP is plotted together with the relevant foregrounds. The SPOrt sensitivities reported in Table 1 confirm that full maps of the Galaxy should be done at 22 and $32 \mathrm{GHz}$, following predictions. 
- to attempt a first detection of CMBP on large angular scales; upper limits, at least 1 order of magnitude lower than at present, can be achieved. Since the SPOrt pixel sensitivities do not envisage the possibility constructing CMBP maps, only full-sky statistical analyses may provide an estimate of the mean polarized signal $P_{\mathrm{rms}}=\sqrt{\left\langle Q^{2}+U^{2}\right\rangle}$. Similarly to what has been done by PIQUE ${ }^{23}$ and POLAR ${ }^{22}$ experiments by applying the flat spectrum analysis, ${ }^{41}$ simulations have shown that the CMBP measurement will have an error $\sigma\left(P_{\mathrm{rms}}\right)=0.3 \mu \mathrm{K}(1 \sigma$ C.L. $)$, taking into account the degradation from foreground subtraction.

Figure 4 of Ref. 42 shows the $P_{\text {rms }}$ behaviour, with respect to $\tau$, is almost independent of other cosmological parameters. That is, the detection of the $P_{\mathrm{rms}}$ on large angular scales is relevant for a clean measurement of the optical depth of the re-ionized medium in the dark ages. A sensitivity of $\Delta \tau=0.13$ for a model with $\tau=0.2$ has been determined through a Fisher matrix analysis.

\section{ACKNOWLEDGMENTS}

We thank V. Natale for useful discussions. We acknowledge use of CMBFAST package for performing our analysis. SPOrt is an ASI funded project. We thank also ESA for provding the flight opportunity onboard the International Space Station. V.A.R., E.N.V., M.V.S. and I.A.S. are greatful to C.N.R. for partially supporting their collaboration within the C.N.R.-R.A.S. agreement.

\section{REFERENCES}

1. Sazhin M. V., Benitez N., Astrophys. Lett. Commun. 32, 105, 1995.

2. Jungman G., Kamionkowski M., Kosowsky A., Spergel D. N., PRD 54, 1332-1344, 1996.

3. Zaldarriaga M., Spergel D. N., Seljak U., ApJ 488, 1, 1997

4. Efstathiou G., Bond J. R., MNRAS 304, 75, 1999.

5. Smoot G.F., et al., ApJ 396, L1, 1991.

6. Bennet C.L., et al., ApJ 464, L1, 1996.

7. De Bernardis P., et al., Nature 404, 955, 2000.

8. Hanany S., et al., ApJ 545, L5, 2000.

9. Miller A. D., et al., ApJ 524, L1, 1999.

10. Kosowsky A., NewAR 43, 157-168, 1999.

11. Rubakov V.A., Sazhin M.V., Veryaskin A.V., Phys. Lett. B 115, pp. 189-192, 1982.

12. Kamionkowski M., Kosowsky A., PRD 57, 685, 1998.

13. Penzias A. A., and Wilson R. W., ApJ 142, 419-221, 1965.

14. Caderni N., et al., Phys. Rev. D 17, 1901-1907, 1978.

15. Nanos G. N., ApJ 232, 341-347, 1979.

16. Lubin P. M., and Smoot G. F., ApJ 245, 1-17, 1981.

17. Partridge R. B., et al., Nature 331, 146-147, 1988.

18. Wollack E. J., et al., ApJ 419, L49-L52, 1993.

19. Netterfield C. B., et al., ApJ 445, L69-L72, 1995.

20. Sironi G., Boella G., Bonelli G., Brunetti L., Cavaliere F., Gervasi M., Giardino G., Passerini A., NewA 3, 1-13, 1998.

21. Subrahmanyan R. et al., MNRAS 315, 808-822, 2000.

22. Keating B. G., et al., ApJ 560, L1, 2001.

23. Hedman M. M., Barkats D., Gundersen J. O., McMahon J. J., Staggs S. T., Winstein B., ApJ 573, L73-L76, 2002.

24. Tucci M., Carretti E., Cecchini S., Fabbri R., Orsini M., Pierpaoli E., NewA 5, 181, 2000.

25. Tegmark M., Eisenstein D. J., Hu W., de Oliveira-Costa A., ApJ 530, 133, 2000.

26. Bruscoli M., Natale V., Tucci M., Carretti E., Fabbri R., Cortiglioni S., Sbarra C., NewA 7, 171, 2002.

27. Brouw W. N., Spoelstra T. A. Th., A\&AS 26, 129, 1976.

28. Duncan A. R., Haynes R. F., Jones K. L., Stewart R. T., MNRAS 291, 279, 1997. 
29. Duncan A. R., Reich P., Reich W., Fürst E., 1999, A\&A 350, 447, 1999.

30. Uyaniker B., Fürst E., Reich W., Reich P., Wielebinski R., AछAS 138, 31, 1999.

31. Gaensler B. M., Dickey J. M., McClure-Griffiths N. M., Green A. J., Wieringa M. H., Haynes R. F., ApJ 549, 959, 2001.

32. Cortiglioni S., et al., in AMiBA 2001: High-z Clusters, Missing Baryons, and CMB Polarization, ASP Conf. Ser. CS-257, in press, 2002

33. Wollack E.J., Rev. Sci. Instrum. 66, 4305, 1995

34. Wollack E.J., Pospieszalski M.W., IEEE MTT-S Digest 1998, p. 669, 1998

35. Tascone R., Trinchero D., Baralis M., Peverini O.A., Olivieri A., Carretti E., Cortiglioni S., in Experimental Cosmology at Millimetre Wavelengths, AIP Conf. Proc. 616, 150, 2002.

36. Peverini O.A., Baralis M., Tascone R., Trinchero D., Olivieri A., Carretti E., Cortiglioni S., in Astrophysical Polarized Backgrounds, AIP Conf. Proc. 609, 177, 2002.

37. Carretti E., Tascone R., Cortiglioni S., Monari J., Orsini M., NewA 6, 173-187, 2001 (astro-ph/0103318).

38. Sbarra C., Carretti E., Cortiglioni S., Zannoni M., Fabbri R., Macculi C., Tucci M., in Astrophysical Polarized Backgrounds, AIP Conf. Proc. 609, 193, 2002.

39. Baralis M., et al., in Astrophysical Polarized Backgrounds, AIP Conf. Proc. 609, 257, 2002.

40. Zannoni M., et al., this volume, 2002.

41. Zaldarriaga M., ApJ 503, 1, 1998.

42. Carretti E., et al., in Astrophysical Polarized Backgrounds, AIP Conf. Proc. 609, 109, 2002. 